Originally published as:

Guo, J.Y., Greiner-Mai, H., Ballani, L., Jochmann, H. and Shum, C.K., 2005, On the doublepeak spectrum of the Chandler wobble, J. Geodesy, 78, 654-659

DOI: $10.1007 / \mathrm{s} 00190-004-0431-0$

http://www.springerlink.com/openurl.asp?genre=article\&eissn=1432-

$\underline{1394 \& \text { volume }=78 \& \text { issue }=11 \text { \&spage }=654}$ 


\title{
On the double-peak spectrum of the Chandler wobble
}

\author{
J. Y. Guo ${ }^{1,2,3}$, H. Greiner-Mai ${ }^{2}$, L. Ballani' ${ }^{2}$, H. Jochmann ${ }^{2}$, C. K. Shum ${ }^{3}$ \\ 1 The Key Laboratory of Geospace Environment and Geodesy, Ministry of Education, School of Geodesy and Geomatics, Wuhan University, \\ 129 Luoyu Road, 430079 Wuhan, China; e-mail: junyiguo@ public.wh.hb.cn; Tel.: +86-27-87851394; Fax: +86-27-68771825 \\ ${ }^{2}$ GeoForschungsZentrum Potsdam, Section 1.3, Telegrafenberg A17, D-14473 Potsdam, Germany; e-mail: grm@ gfz-potsdam.de, bal@ gfz- \\ potsdam.de, h.jochmann@t-online.de; Tel.: +49-331-2881144(HGM), +49-331-2881142(LB), +49-3328-304798(HJ); Fax: +49-331-2881163 \\ 3 Laboratory for Space Geodesy and Remote Sensing Research, The Ohio State University, 2070 Neil Avenue, Columbus, Ohio 43210-1275, \\ USA; e-mail: ckshum@ osu.edu, guo.81@ osu.edu; Tel.: +1-614-292-7118(CKS), +1-614-292-2957(JYG); Fax: +1-614-292-2957
}

Received: date / Revised version: date

\begin{abstract}
It is well known that two close spectral peaks with comparable amplitudes exist in the Chandler wobble (CW) band in the observed polar motion spectrum during the first half of the past century. However, it is not generally accepted that the $\mathrm{CW}$ is an oscillation with two closely lying frequencies, and some authors attribute the appearance of these two peaks to some unusual phase variation $\left(180^{\circ}\right.$ shift) of the CW during $1920 \mathrm{~s}-1930 \mathrm{~s}$. In this note, the phase variation of the $\mathrm{CW}$ is explored using the longest Earth orientation parameter (EOP) time series currently available - the International Earth Rotation and Reference System Service (IERS) EOP-C01 from 1846 to 2003. The observation time series mainly containing the CW obtained by removing the annual wobble (AW) from the original EOP time series is compared with a synthetic double frequency CW times series constructed using the frequencies, amplitudes and initial phases of the two peaks precisely determined using spectral analysis. The result shows reasonable agreement between their peak times, a feature not shown before. This feature is in agreement with the hypothesis of a double frequency $\mathrm{CW}$. While the result shown here is inconclusive that the $\mathrm{CW}$ really has two frequencies, this note is expected to serve as a reminder for its renewed interest.
\end{abstract}

Key words. Chandler wobble - Double frequency - Phase shift

\section{Introduction}

The Chandler wobble $(\mathrm{CW})$ is well known to be one of the most important and enigmatic components of polar motion. Spectral analysis of the polar motion time series during the first half of the past century revealed two closely lying spectral peaks with comparable amplitudes in the $\mathrm{CW}$ frequency band, and some authors attempted to interpret this in terms of a double frequency CW (e.g., Labrouste and Labrouste 1946; Yashkov 1965; Gaposchkin 1972). Colombo and Shapiro (1968) tried to provide a theoretical model for a double frequency CW. Chao (1983) argued that, from the point of view of numerical analysis rather than geophysical interpretation, the then International Latitude Service (ILS) data support the multiple component hypothesis of the $\mathrm{CW}$, and used four components to represent the polar motion in the $\mathrm{CW}$ band.

However, some authors showed that the appearance of the two peaks does not necessarily imply a double frequency CW from a statistical point of view (e.g., Pedersen and Rochester 1972; Lenhardt and Groten 1985; Okubo 1982), and that the double peak feature could generally be explained based on a single frequency oscillation. One such explanation is that the phase of the CW has been subjected to some unusual variation (a phase shift of $180^{\circ}$ ) during 1920s-1930s (Fedorov and Yatskiv 1965; Guinot 1972; McCarthy 1974; Dickman 1981; Vondrák 1985). Another explanation is to accept a variable CW frequency. In fact, the variability of the $\mathrm{CW}$ frequency has been a subject of debate. Some authors assumed or proved the frequency to be invariant (e.g., Ooe 1978; Kuehne and Wilson 1996; Vicente and Wilson 1997; Gross et al. 2003; Liao et al. 2003 ; Seitz et al. 2004). Some authors suggested a variable frequency (e.g., Melchior 1954, 1957; Sekiguchi 1972, 1976; Guinot 1972; Carter 1981; Nastula et al. 1993; Liu et al. 2000; Schuh et al. 2001; Höpfner 2003, 2004). Jochmann (2003) argued that, as the CW is a proper mode of the Earth, the variation of its frequency should depend on the variation of the dynamical structure of the Earth, and showed that the variation due to changes in the structure of the Earth is insignificant. Here we accept this opinion and ignore the possible explanation in terms of frequency variation.

In this note, we examine how the phase of the CW has shifted during the 1920s-1930s using the longest Earth orientation parameter (EOP) time series currently available: the International Earth Rotation and Reference System Service (IERS) EOPC01 from 1846 to 2003. Special attention is focused on a comparison between the observed CW obtained by removing the annual wobble (AW) from the EOP time series and a double frequency synthetic CW time series built with the frequencies, 
amplitudes and initial phases of the two peaks precisely determined by spectral analysis. The result shows reasonable agreement between their peak times, a feature not shown before. We do not try to conclude here that the $\mathrm{CW}$ is a double frequency oscillation based on this agreement alone. However, we suggest nevertheless that this double peak feature is not necessarily due to numerical artifacts, and merits further investigation for understanding its geophysical cause.

\section{The double-peak spectral property}

We use essentially the same spectral analysis method as Yashkov (1965). This method was re-studied by Guo et al. (2004) for precisely determining the frequency of periodical oscillation using a time series of observations, and was referred to as the folding-averaging algorithm (FAA). The basic principle of the FAA is to rebuild for every test period a new short time series by cutting the original time series to shorter ones of equal length identical to the test period, and then averaging them. In the averaged short time series, the amplitude of the possible signal with a period equal to the test period remains the same, but signals of different periods are averaged out and the random error is reduced. The amplitude and phase of the possible signal with a period equal to the test period can then be estimated using the averaged short time series. By searching for the extremes of the amplitude by varying the test period, the periods (and the amplitudes and phases) of the signals which may be present in the time series can be precisely determined. See the Electronic Supplementary Material (ESM) of this paper for detail.

In the IERS EOP-C01 time series, the data are in 0.1 year intervals during 1846.0 to 1890.0 , and in 0.05 year intervals during 1890.0 to 2004.0. We have adopted the interval of 0.1 year by dropping one data over two during 1890.0 to 2004.0. We have also removed the long term variation that is estimated by spline interpolation of the non-overlapping means of 13 years (about twice the length of the beating period between the CW and AW) (Jeffreys 1968; Vicente and Wilson 1997).

First we compute the amplitude spectra for the components $X_{p}$ and $Y_{p}$ by using the FAA to an array of selected frequency/period (Guo et al. 2004; See also the ESM), that is similar to the fast Fourier transform (FFT) with zero padding. These spectra are then used to construct the spectrum for the complex-valued EOP time series $\tilde{P}=X_{p}-i Y_{p}$. We express the harmonic signals with an angular frequency $\omega$ in the components $X_{p}$ and $Y_{p}$ in the form of

$X_{p}=A_{x} \sin \left(\omega t+\phi_{x}\right), \quad Y_{p}=A_{y} \sin \left(\omega t+\phi_{y}\right)$.

They correspond to a prograde-retrograde pair of harmonic signals in the complex-valued EOP time series $\tilde{P}$ :

$\tilde{P}=A_{+} e^{i\left(\omega t+\phi_{+}\right)}+A_{-} e^{-i\left(\omega t+\phi_{-}\right)}$.

It can be shown that

$$
\begin{array}{r}
A_{+} \cos \phi_{+}=\left(A_{x} \sin \phi_{x}-A_{y} \cos \phi_{y}\right) / 2, \\
A_{+} \sin \phi_{+}=-\left(A_{x} \cos \phi_{x}+A_{y} \sin \phi_{y}\right) / 2, \\
A_{-} \cos \phi_{-}=\left(A_{x} \sin \phi_{x}+A_{y} \cos \phi_{y}\right) / 2, \\
A_{-} \sin \phi_{-}=-\left(A_{x} \cos \phi_{x}-A_{y} \sin \phi_{y}\right) / 2 .
\end{array}
$$

These are the basic formulas for computing the spectrum of $\tilde{P}$ using the spectra of $X_{p}$ and $Y_{p}$. We show in Fig. 1 the amplitude spectrum in the AW and CW band. A similar diagram may be found in Pedersen and Rochester (1972) (Fig. 1) who used the FFT, and more recently Jochmann and Felsmann (2001) (Fig. 1) who used a modified version of the Fourier transform to obtain a very clean and sharp peak spectrum.

Figure 1 near here

Table 1 near here

The precise frequencies of the wobbles are first determined by extensively searching for the maximum amplitudes using a very dense test frequency/period near the peaks found in the spectrum (Fig. 1). The amplitudes and phases are then estimated by applying the least squares method to the average short time series built according to the precise values of frequencies of the wobbles (Guo et al. 2004; See also the ESM). We adopt here an iterative process to achieve additional accuracy. At each iteration, before building the averaged short time series for estimating the amplitude and phase for one wobble, the other wobbles are first removed from the original time series using their amplitude and phase estimated in the previous iteration. In this step, the amplitudes and phases of the spectrum of the complex-valued EOP time series $\tilde{P}$ are also determined using the amplitudes and phases of the spectra of the components $X_{p}$ and $Y_{p}$ according to Eq. (3)-(6). The frequencies, amplitudes and initial phases of the AW and the two peaks in the $\mathrm{CW}$ band (denoted with $\mathrm{CW}_{1}$ and $\mathrm{CW}_{2}$ ) precisely determined are listed in Table 1. As the annual wobble is only slightly elliptical (Vondrák 1985), we approximately treat it as a circular mode. Our estimates of the two frequencies in the CW band are 0.8495 and 0.8400 cycle per year (cpy) (the periods are, respectively, 1.1771 and 1.1905 years or 429.9 and 434.8 days). The difference between the two frequencies in the CW band is about 0.01 cpy, giving a beating period of about 100 years.

It is generally accepted that the AW is relatively stable over time (e.g., Pedersen and Rochester 1972; Vondrák 1985, 1999; Höpfner 2004). Considering the fact that the AW and the CW are the most important constituents of polar motion, for both the 
components $X_{p}$ and $Y_{p}$, by removing the AW as a simple harmonic signal according to the parameters listed in Table 1, we obtain a time series that principally contains the $\mathrm{CW}$, which will be loosely called the observed $\mathrm{CW}$. As the annual wobble is only slightly elliptical (Vondrák 1985), removing it as a circular mode is not expected to distort the result. We also construct a synthetic time series composed of two harmonic waves for both the components $X_{p}$ and $Y_{p}$ using the parameters of the two peaks in the CW band as listed in Table 1, which will be referred to as the double frequency synthetic CW. As an example, we show a comparison between the observed CW and the double frequency synthetic CW for the component $X_{p}$ in Fig. 2 (The figure for the component $Y_{p}$ is similar. It is provided in the ESM).

Figure 2 near here

We begin to discuss Fig. 2 by considering the properties of the sum of two harmonic signals of very close frequencies and amplitudes, i.e. the two peaks in the CW band shown in Table 1 . We take again the component $X_{p}$ as example, and write

$X_{p}=A_{1} \sin \left(\omega_{1} t+\phi_{1}\right)+A_{2} \sin \left(\omega_{2} t+\phi_{2}\right)$

where we assume, according to the data listed in Table 1 ,

$\begin{array}{ll}\omega_{1}>\omega_{2}, & \omega_{1}-\omega_{2}<<\omega_{1} \text { and } \omega_{2} \\ A_{1}>A_{2}, & A_{1}-A_{2}<<A_{1} \text { and } A_{2}\end{array}$

For a simple interpretation, we rewrite Eq. (7) in the form

$X=2 A_{2} \cos \left(\omega^{\prime} t+\phi^{\prime}\right) \sin (\omega+\phi)+\Delta A \sin \left(\omega_{1} t+\phi_{1}\right)$

where

$$
\begin{gathered}
\Delta A=A_{1}-A_{2}, \\
\omega=\left(\omega_{1}+\omega_{2}\right) / 2, \quad \omega^{\prime}=\left(\omega_{1}-\omega_{2}\right) / 2, \\
\phi=\left(\phi_{1}+\phi_{2}\right) / 2, \quad \phi^{\prime}=\left(\phi_{1}-\phi_{2}\right) / 2 .
\end{gathered}
$$

In Eq. (10), the first term at the right hand side is equal to $A_{2} \sin \left(\omega_{1} t+\phi_{1}\right)+A_{2} \sin \left(\omega_{2} t+\phi_{2}\right)$ that is the sum of two harmonic signals. So this term can be understood either as a double frequency signal or a single frequency signal with modulated amplitude and a phase shift of $180^{\circ}$ at around $\omega^{\prime} t+\phi^{\prime}= \pm \pi / 2$ where it vanishes. From this point of view, it is not possible to distinguish between a single frequency signal and a double frequency signal with only one phase shift of $180^{\circ}$.

At a time span not covering the vicinity of $\omega^{\prime} t+\phi^{\prime}= \pm \pi / 2$, Eq. (10) is dominated by the first term, thus behaving like a single frequency signal with modulated amplitude; its frequency is $\omega$ defined in Eq. (12) that has a numerical value of 0.8448 cpy, corresponding to a period of 1.1838 years (432.4 days). In Fig. 2, the distance between the vertical grid lines is just this period. We see that before 1920, the grid lines coincide with the higher peaks of both the observed CW and the double frequency synthetic CW, and after 1940, at the lower peaks. If we interpret both the observed CW and the double frequency synthetic CW as a single frequency signal with phase shift, this property indicates that there is a phase shift of $180^{\circ}$ during 1920-1940. Furthermore, it also indicates that the peak times of the observed CW and the double frequency synthetic CW are in good agreement before 1920 and after 1940; the peak-to-peak distance being around the period of the CW if it is considered as a single frequency wobble, i.e. 1.1838 years.

The phase shift, which may be understood as the peak-to-peak distance variation, can be seen in more detail in the vicinity of $\omega^{\prime} t+\phi^{\prime}= \pm \pi / 2$, i.e. during 1920-1940, where the influence of the second term is also important. In general, we see from Fig. 2 that, during the period 1920-1940, the phase has gradually shifted $180^{\circ}$ in advance for both the observed CW and the double frequency synthetic $\mathrm{CW}$ if we interpret both of them as single frequency signals with phase shift. We also notice that the peak times of the observed $\mathrm{CW}$ and the double frequency synthetic $\mathrm{CW}$ are in reasonably good agreement, considering the small amplitude of the $\mathrm{CW}$ and the presence of error of observation and variations with shorter periods not removed from the observed CW that we obtained by removing only the annual wobble from the EOP time series. This agreement may in fact be understood as a fact supporting the hypothesis of a double frequency $\mathrm{CW}$, though not sufficient to draw a conclusion for its definite existence.

Particularly, during the short time nearest to $\omega^{\prime} t+\phi^{\prime}= \pm \pi / 2$, i.e. around 1930, Eq. (10) is dominated by the second term that has a frequency $\omega_{1}$, slightly higher than $\omega$ discussed in the last paragraph. The peak-to-peak distance at this time is just the period corresponding to the higher frequency $\omega_{1}$, the numerical value being 1.1771 years (429.9 days). This is, in fact, the smallest peak to peak distance (at least for the double frequency synthetic $\mathrm{CW}$ ).

To see the phase shift in more detail, we give an alternative expression of (7) (i.e., again only considering the component $\left.X_{p}\right)$ :

$X_{p}=A(t) \sin [\omega t+\phi+\beta(t)]$

where the amplitude $A(t)$ and the phase shift terms $\beta(t)$ are

$A(t)=\left[A_{1}^{2}+A_{2}^{2}+2 A_{1} A_{2} \cos \left(2 \omega^{\prime} t+2 \phi^{\prime}\right)\right]^{1 / 2}$, 
$\beta(t)=\arctan \left[\frac{A_{1}-A_{2}}{A_{1}+A_{2}} \tan \left(\omega^{\prime} t+\phi^{\prime}\right)\right]$.

Eqs. (14)-(16) express the sum of two sinusoidal signals in terms of only one with amplitude and phase modulated. The phase shift is given by (16). From these expressions, we again see that the amplitude is smallest near $\omega^{\prime} t+\phi^{\prime}= \pm \pi / 2$. It can also been seen that the phase-shift term $\beta(t)$ varies fastest at these locations, since $\left(A_{1}-A_{2}\right) /\left(A_{1}+A_{2}\right)$ is of very small order of magnitude, only 0.11 according to the data given in Table 1 . For example, $\beta(t)$ varies from 0 to $1 / 5 \times \pi / 2$ and then to $\pi / 2$ when $\omega^{\prime} t+\phi^{\prime}$ varies from 0 to $4 / 5 \times \pi / 2$ and then to $\pi / 2$. Although Eqs. (14)-(16) give accurate phase shift, they are not as simple as Eq. (10) when examining the peak-to-peak distance during the short time nearest to $\omega^{\prime} t+\phi^{\prime}= \pm \pi / 2$, as discussed in the last paragraph.

\section{Discussion}

We have compared the observed $\mathrm{CW}$ and the double frequency synthetic CW built using the parameters of the two peaks in the CW band precisely determined using spectral analysis (Table 1). We have focused on the period around 1930 when a phase shift of $180^{\circ}$ responsible for the appearance of the double peak in the $\mathrm{CW}$ band is believed to have happened. The peak times of the observed CW and the double frequency synthetic CW agree reasonably well considering the small amplitude of the CW during that period and the presence of error of observation and variations with shorter periods that we have not removed from the observed $\mathrm{CW}$ we have defined. This agreement supports the hypothesis of a double frequency $\mathrm{CW}$, though not enough to draw a definitive conclusion.

The CW is a free mode of the Earth, which has been decaying and re-excited. Such a free mode cannot be expected to be purely harmonic; amplitude variation and phase shift are natural. The large phase shift during 1920s-1930s may well be attributed to the excitation process while adopting a single frequency CW (e.g, Jeffreys 1940; Vondrák 1999). However, a phase shift of $180^{\circ}$ during a very short time is something peculiar, though the small amplitude of the CW during that time may render a large phase shift to be caused more easily by the excitation process. Guinot (1982) pointed out that the cause of the event is probably quite different from the excitation which modifies the amplitude. Examination of the process of this large phase shift may potentially be helpful for understanding the cause of the event.

One may argue that the double peak feature of the CW is not real because only one peak appears in the spectra of the EOP time series before 1920, after 1940, or between 1920 and 1940 (e.g., Pedersen and Rochester 1972; Lenhardt and Groten 1985). We have computed the spectra for the time series of the double frequency synthetic CW during 1846-1920, 1940-2003 and 1920-1940 respectively. The double peak feature did not appear in the resulting spectra, although the time series really contain two harmonic signals. This implies that, even if the CW really has two frequencies, one can only expect to obtain them by spectral analysis of an EOP time series long enough and including the period around 1930 when the variation of the CW is most dramatic. Thus, we believe that the results of spectral analysis of a short EOP time series, or an EOP time series not covering the period around 1930 cannot be used to deny the possibility of a double frequency CW.

The comparison made in this paper is qualitative. No statistical test has been made. In fact, it is not evident that certain firm criteria may be found to really judge if the $\mathrm{CW}$ is single or double frequency. As already mentioned, the $\mathrm{CW}$ is not purely harmonic. So it is basically inappropriate to describe a single or double frequency CW with 3 or 6 parameters (frequency, amplitude and initial phase). Although we believe that the correlation between the phases of the observed CW and the synthetic double frequency $\mathrm{CW}$ is in favor of a double frequency $\mathrm{CW}$, we do not think that a definite conclusion may be drawn based on this correlation alone. In summary, we suggest that, although it is generally believed that the EOP data available up to now may be explained using a single frequency $\mathrm{CW}$, it seems that the possibility of a double frequency CW could not definitely be ruled out. We also suggest that the definitive answer may only be drawn in about 30 years to see whether another variation similar to that around 1930 will happen again, since the beat time between the two frequencies in the CW band is about 100 years.

The main reason for which the idea of a double frequency $\mathrm{CW}$ is not accepted is that there exists no reliable theoretical model of the free wobble of the Earth based on the Earth's shape and internal constitution which predict this phenomena. Recently, Rogister and Vallete (2004) proposed a wobble model of the Earth with non-neutrally stratified outer core capable of producing two wobbles at the CW band with periods 2-5 days apart assuming equal amplitudes. As compared to this model, the minor difference in the amplitudes of the two peaks in the CW band obtained from the EOP time series in this paper is insignificant, while the difference between the periods of these two peaks is 5 days, that is within the limit of the model. However, this coincidence is still insufficient to conclude that the $\mathrm{CW}$ really has two frequencies. Excitation based on the model of Rogister and Vallete (2004) need to be further studied. This is evidently another work.

Furthermore, besides a phase shift that is generally accepted, a double frequency CW may not be the only explanation of the double peak. Perhaps some nonlinearity may make the $\mathrm{CW}$ behaves in a more complicated way that appears like a double frequency harmonic signal.

In addition to the comparison of phases, we expect this note to serve as a reminder for the double frequency feature of the EOP time series in the CW band for potential renewed interest in explaining this peculiar phenomena.

Acknowledgment We gratefully acknowledge the editor-in-chief, Prof. Featherstone, and Prof. Vondrák and two anonymous reviewers for valuable comments that lead to various improvements of the manuscript. J. Y. Guo is supported by the Natural 
Science Foundation of China under grants 40274028 and the Excellent Young Teachers Program of Ministry of Education of China.

\section{References}

Carter WE (1981) Frequency modulation of the Chandlerian component of polar motion. J Geophys Res 86(B3): 1653-1658

Chao BF (1983) Autoregressive harmonic analysis of the Earth's polar motion using homogeneous ILS data. J Geophys Res 88(B12): 1029910307

Colombo G, Shapiro II (1968) Theoretical model for the Chandler wobble. Nature 217: 156-157

Dickman SR (1981) Investigation of controversial polar motion features using homogeneous International Latitude Service data. J Geophys Res 86(B6): 4904-4912

Fedorov EP, Yatskiv YS (1965) The cause of the apparent "bifurcation" of the free nutation period. Soviet Astron 8: 608-611

Gaposchkin EM (1972) Analysis of pole position from 1846 to 1970. In: Melchior P, Yumi S (eds) Rotation of the Earth. Reidel, Dordrecht, pp 33-38

Gross RS, Fukumori I, Menemenlis D (2003) Atmospheric and oceanic excitation of the Earth's wobbles during 1980-2000. J Geophys Res 108(B8): 2370, doi:10.1029/2002JB002143

Guinot B (1972) The Chandlerian wobble from 1900 to 1970. Astron Astrophys 19: 207-214

Guinot B (1982) The Chandlerian nutation from 1900 to 1980. Geophys J R astr Soc 71(2): 295-301

Guo JY, Greiner-Mai H, Dierks O, Ballani L, Neumeyer J, Shum CK (2004) The folding-averaging algorithm for precisely determining periods of signals in time series with application to the study of the Earth's free oscillations using superconducting gravimeter data. Phys Earth Planet Inter: Submitted

Höpfner J (2003) Chandler and annual wobbles based on space-geodetic measurements. J Geodyn 36: 369-381

Höpfner J (2004) Low-frequency variations, Chandler and annual wobbles of polar motion as observed over one century. Surveys in Geophysics 25: $1-54$

Jeffreys H (1940) The variation of latitude. Mon Not R Astron Soc 100: 139-155

Jeffreys H (1968) Thye variation of latitude. Mon Not R Astron Soc 141, 255-268.

Jochmann H, Felsmann E (2001) Evidence and cause of climate cycles in polar motion. J Geod 74: $711-719$

Jochmann H (2003) Period variations of the Chandler wobble. J Geod 77: 454-458

Kuehne J, Wilson CR (1996) Estimates of the Chandler wobble frequency and Q. J Geophys Res 101(B6): 13 537-13 579

Labrouste H, Labrouste Y (1946) Composantes périodiques de la variation des latitudes. Annales de Géophysique 2: 115-130

Lenhardt H, Groten E (1985) Chandler wobble parameters from BIM and ILS data. manuscr geod 10: 296-305

Liao D, Liao X, Zhou Y (2003) Oceanic and atmospheric excitation of the Chandler wobble. Geophys J Int 152: 215-227, doi:10.1111/j.1365246X.2004.02208.X

Liu LT, Hsu HT, Gao BX, Wu B (2000) Wavelet analysis of the variable Chandler wobble. Geophys Res Lett 27(18): 3001-3004

McCarthy D (1974) The variation of latitude based on U. S. Naval Observatory photographic zenith tube observations. J Geophys Res 79: 3343-3346

Melchior P (1954) Contribution à l'étude des mouvements de l'axe instantané de rotation par raport au globe terrestre. Monograhies 3, Observatoire Royal de Belgique. Impr R Louis, Ixelles-Bruxelles

Melchior P (1957) Latitude variation. In: Physics and chemistry of the Earth, Vol. 2, pp 212-243. Pergamon Press, Oxford, New York

Nastula J, Korsun A, Kolaczek B, Kosek W, Hozakowski W (1993) Variation of the Chandler and annual wobbles of polar motion in 18461988 and their prediction. manuscr geod 18: 131-135

Okubo S (1982) Is the Chandler period variable? Geophys J R astr Soc 71: 629-646

Ooe M (1978) An optimal complex ARMA model of the Chandler wobble. Geophys J R astr Soc 53: 445-457

Pedersen GPH, Rochester MG (1972) Spectral analysis of the Chandler wobble. In: Melchior P, Yumi S (eds) Rotation of the Earth. Reidel, Dordrecht, pp 33-38

Rogister Y, Valette B (2004) Influence of outer core dynamics on Chandler wobble period. Chandler Wobble Workshop, European Center for Geodynamics and Seismology, Luxembourg, April 21-23, 2004

Schuh H, Nagel S, Seitz T (2001) Linear drift and periodic variations observed in long term series of polar motion. J Geod 74: 701-710

Seitz F, Stuck J, Thomas M (2004) Consistent atmospheric and oceanic excitation of the Earth's free polar motion. Geophys J Int 157(1): 25-35

Sekiguchi N (1972) On some properties of the excitation and damping of the polar motion. Publ Astron Soc Japan 24: 99-108

Sekiguchi N (1976) An interpretation of the multiple-peak spectra of the polar wobble of the Earth. Publ Astron Soc Japan 28: 277-291

Vicente RO, Wilson CR (1997) On the variability of the Chandler frequency. J Geophys Res 102(B9): 20 439-20 445

Vondrák J (1985) Long-period behaviour of polar motion between 1900.0and 1984.0. Annales Geophysicae 3: $351-356$

Vondrák J (1999) Earth Rotation Parameters 1899.7-1992.0 After Reanalysis Within The Hipparcos Frame. Surveys in Geophysics 20: 169195

Yashkov VY (1965) Spectrum of the motion of the Earth's poles. Soviet Astron 8: 605-607 


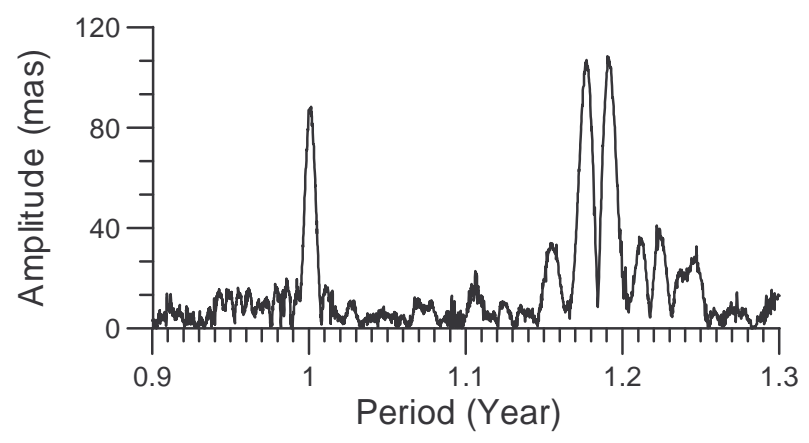

Fig. 1. The amplitude spectrum in the AW and CW band computed using the IERS EOP-C01 time series during 1846-2003, which shows the double peak of the CW. 


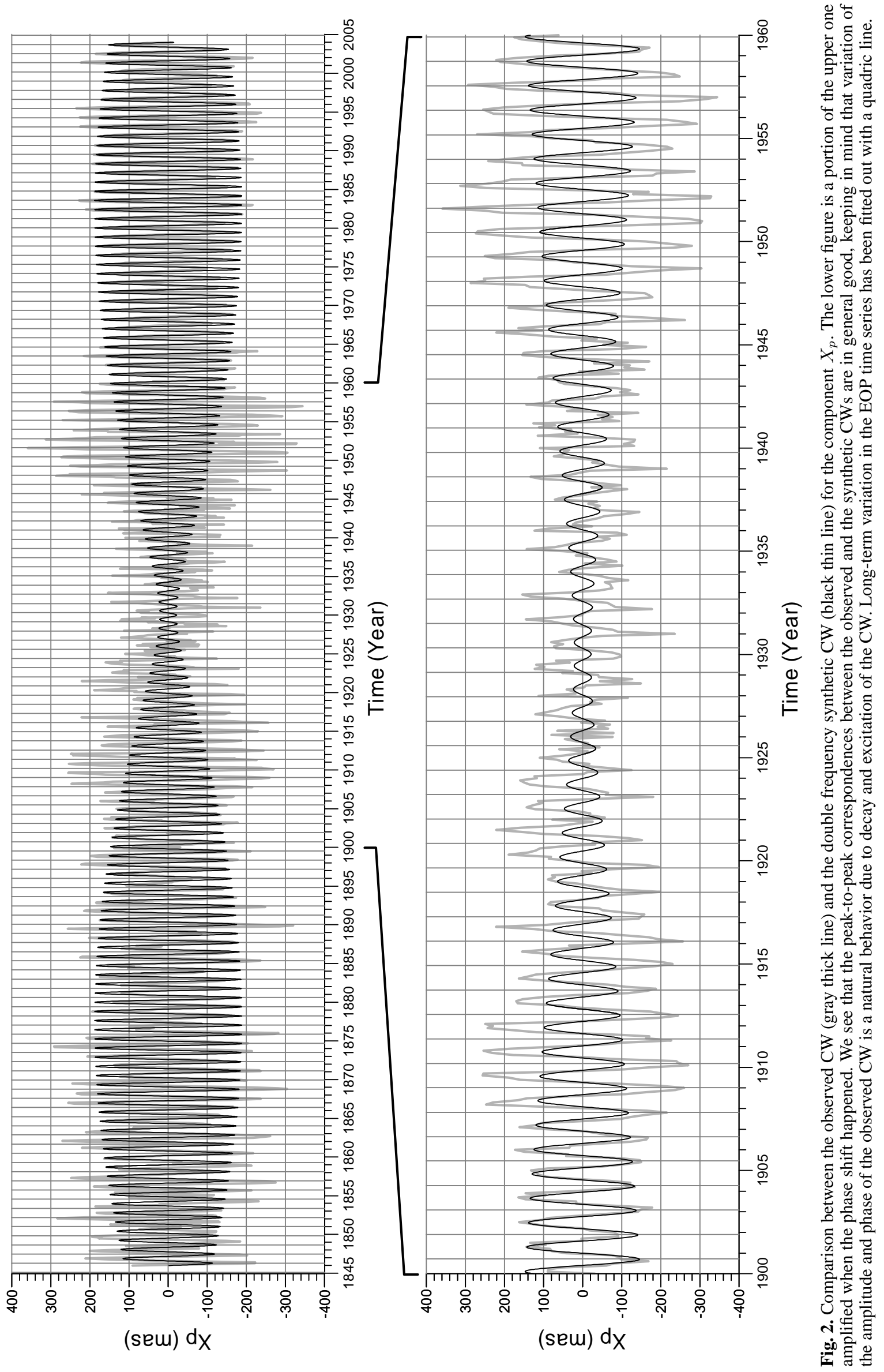


Table 1. Periods, amplitudes and initial phases of the AW and the two peaks in the CW band. Initial time: 1846.0.

\begin{tabular}{lccc}
\hline Wobble & $\begin{array}{c}\text { Frequency } \\
\text { cycle/year }\end{array}$ & $\begin{array}{c}\text { Amplitude } \\
\text { mas }\end{array}$ & $\begin{array}{c}\text { Initial phase } \\
\left({ }^{\circ}\right)\end{array}$ \\
\hline $\mathrm{AW}$ & 0.99866 & 88.540 & 166.73 \\
\hline $\mathrm{CW}_{1}$ (First peak) & 0.84953 & 104.759 & 45.89 \\
\hline $\mathrm{CW}_{2}$ (Second peak) & 0.84001 & 83.333 & 153.91 \\
\hline
\end{tabular}

